\title{
Survival in Frontotemporal Dementia Phenotypes: A Meta-Analysis
}

\author{
Kalyani Kansala Manisha Mareddy ${ }^{\mathrm{a}}$ Kelly L. Sloane ${ }^{a, b} \quad$ Alexa A. Minc $^{c}$ \\ Peter V. Rabins ${ }^{a}$ John B. McGready ${ }^{d}$ Chiadi U. Onyike ${ }^{a}$ \\ ${ }^{a}$ Division of Geriatric Psychiatry and Neuropsychiatry, Department of Psychiatry and \\ Behavioral Sciences, and bepartment of Neurology, Johns Hopkins University School \\ of Medicine, Baltimore, Md., ' Health and Human Behavior Program, Brown University, \\ Providence, R.I., and d Department of Biostatistics, Johns Hopkins Bloomberg School of \\ Public Health, Baltimore, Md., USA; eJSS Medical College, Mysore, India
}

\section{Key Words}

Frontotemporal dementia - Semantic dementia - Progressive non-fluent aphasia · Progressive supranuclear palsy · Corticobasal degeneration · Epidemiology · Survival · Life expectancy · Years of life lost

\begin{abstract}
Background: Survival in frontotemporal dementia (FTD) is not well understood. We conducted a mixed effects meta-analysis of survival in FTD to examine phenotype differences and contributory factors. Methods: The PubMed, Medline, EMBASE, CINAHL, PsycINFO and Cochrane databases were searched for studies describing survival or natural history of behavioral variant FTD (bvFTD), progressive non-fluent aphasia (PNFA), semantic dementia (SD), FTD with amyotrophic lateral sclerosis (FTD-ALS), progressive supranuclear palsy and corticobasal degeneration. There were no language restrictions. Results: We included 27 studies (2,462 subjects). Aggregate mean and median survival were derived for each phenotype and, for comparison, Alzheimer's disease (AD) (using data from the selected studies). Survival was shortest in FTD-ALS (2.5 years). Mean survival was longest in bvFTD and PNFA (8 years) and median survival in SD (12 years). AD was comparable in survival to all except FTD-ALS. Age and sex did not affect survival; the education effect was equivocal. Heterogeneity in FTD survival was largely, but not wholly, explained by phenotypes. Conclusions: Survival differs for FTD phenotypes but, except for FTD-ALS, compares well to AD survival. Elucidating the potential causes of within-phenotype heterogeneity in survival (such as complicating features and comorbidities) may open up opportunities for tailored interventions.

(C) 2016 S. Karger AG, Basel
\end{abstract}




\section{Introduction}

Frontotemporal dementia (FTD) is, along with Alzheimer's disease (AD), a leading cause of mid-life dementia. It is heterogeneous, encompassing several syndromes that differ in their signal cognitive, behavioral, language and motor phenomena: (1) Behavioral variant FTD (bvFTD), characterized by progressive disintegration of temperament, judgment and conduct. (2) Two types of primary progressive aphasia (PPA): progressive non-fluent aphasia (PNFA), characterized by non-fluent, effortful and agrammatic speech, and semantic dementia (SD), which features fluent speech, with anomia and agnosia for words and objects. Logopenic aphasia, a third PPA type, is usually an AD variant [1]. (3) FTD with amyotrophic lateral sclerosis (FTD-ALS), in which the dementia is associated with progressive paralysis. (4) Progressive supranuclear palsy (PSP), featuring oculomotor palsy, executive dysfunction, behavioral dyscontrol, parkinsonism and postural imbalance. (5) Corticobasal degeneration (CBD), featuring cognitive dysfunction, facial and oropharyngeal apraxia as well as asymmetric limb apraxia, parkinsonism, myoclonus and dystonia.

The life expectancy in FTD is still not clear. A 2011 summary [2] identified a range of 3 14 years from illness onset (i.e., the putative time at which the earliest symptom signifying the illness appeared), varying with phenotype and differing between clinical cohorts (which used prospective observations) and autopsy samples (which were retrospective). The median survival was shown to be 7-13 years in clinical cohorts and 6-8 years in autopsy series, but these observations were not a quantitative analysis of the extant data and did not extend to all phenotypes. Some studies have indicated that FTD-ALS has a particularly poor prognosis, with a survival of 2-3 years [3-5]; however, survival reaches 5 years in some subjects [6]. How other phenotypes compare to one another is not clear, as there have been few relevant analyses. Two survival studies suggested that SD has longer median survival than bvFTD [5, 7], but direct comparisons of survival curves yielded mixed results [4, 8]. Also, we do not know how demographic characteristics, clinical features complicating the illness (e.g., parkinsonism and dysphagia) and comorbid conditions (e.g., heart disease, cancer, diabetes mellitus) shape FTD survival.

There is a common perception that FTD has shorter survival than AD, but quantitative comparisons reached differing conclusions [7, 9-11]. A semi-quantitative review [12] concluded that AD has slightly better survival from diagnosis, with premature mortality (as years of life lost, YLL) greater in FTD and vascular dementia. However, just seven studies were considered (a subset of all with FTD survival data), survival from illness onset was not addressed, and FTD phenotypes were not compared.

To address some of the outstanding issues, we conducted a meta-analysis of survival from illness onset and YLL in all FTD phenotypes. We made comparisons between phenotypes and with $\mathrm{AD}$, and examined whether subject characteristics (age, sex and education) influence survival. The meta-analysis was based on studies that specified phenotype (regardless of autopsy validation), since we sought estimates that can readily be translated to clinical practice (prognostication and treatment), rehabilitative care, community services, epidemiological and interventional research, and policymaking.

\section{Methods}

Literature Search

Three investigators, K.K., M.M. and K.L.S., independently searched the PubMed, Medline, EMBASE, CINAHL, PsycINFO and Cochrane databases with the terms 'frontotemporal dementia', 'frontotemporal lobar degeneration', 'FTD', 'FTLD', 'primary progressive aphasia', 'progressive non-fluent aphasia', 'semantic dementia', 'corticobasal degeneration' or 'progressive supranuclear palsy', combined with 'mortality', 'sur- 
vival', 'prognosis', 'death', 'natural history' or 'life expectancy'. The latest search was conducted on January 5, 2015. The search focused on human subjects research, with no language limitation. The emphasis was titles or abstracts describing survival, natural history or disease progression in FTD phenotypes. We selected original data quantifying illness duration (specified or calculable means or medians with standard errors) and excluded reviews, single case reports and family studies. Two non-English papers were translated with Google Translate (one did not make it to the final set) and one by a native speaker. Family studies, being highly selected samples in which mutation carriers are overrepresented, would, if included, bias the aggregate estimates and preclude their generalizability. References were scoured for more studies fitting the criteria. For two studies $[8,10]$, the authors provided original data. When papers shared samples, the larger studies were selected. The final selection was 27 papers (online suppl. fig. 1 shows a flowchart of the selection strategy; for all online suppl. material, see www.karger.com/doi/10.1159/000443205).

\section{Diagnostic Criteria}

Many diagnostic criteria were used during the 40-year span the studies covered (online suppl. table 1). The 2011 Rascovsky criteria [13], the 1998 Neary criteria [14] and the 1994 Lund-Manchester criteria [15] were used for bvFTD. The Neary criteria and Mesulam's 1987 description [16] were applied to PNFA. SD diagnoses fit the Neary [14] or Gorno-Tempini criteria [1]. One study used Mesulam's 2001 criteria [17] for PPA. PSP diagnoses were based on the original description [18] and four sets of criteria [19-22], except in four studies (two had autopsy validation and one a compelling clinical description). CBD diagnosis was based on three sets of criteria [23-25]; one of the two studies that did not use formal criteria provided autopsy validation. For FTD-ALS, just two studies used formal criteria: one the revised El Escorial criteria [26] for ALS, the other the 2009 Strong criteria for cognitive and behavioral impairment in ALS [27].

\section{Statistics}

Survival, YLL, age at illness onset (defined as the age at which the first symptom appeared), sex, education, sampling frame (recruitment method, center and time period), phenotype and autopsy status were extracted from all studies by two investigators (K.K. and M.M.) independently. AD data were derived from disease control groups in the selected studies. YLL was estimated from mean and median survival (YLLmean and YLL-median, respectively), using country-, gender- and age-specific life expectancy tables that matched the estimated mean year of onset - from the Human Life-Table Database (www.lifetable.de), the WHO life tables (http://www.who.int/gho/mortality_burden_disease/life_tables/life_tables/en/), the Canadian Human Mortality Database (http://www.bdlc.umontreal.ca/chmd/archives.htm) and the Census of India website (http://www.censusindia.gov.in/vital_statistics/SRS_Based/SRS_Based.html). The procedure is described in online supplementary box 1.

Meta-analytic mixed effects regression was implemented with the R package 'metafor' [28], with survival or YLL the dependent variable and phenotypes and demographic factors the fixed moderators. Mixed effects modeling accounted for (1) variability in observed moderators (fixed effects), (2) variability in unobserved study characteristics (residual heterogeneity in the reference populations, accounted for by random effects) and (3) intrinsic variations in study populations (sample variance). A single random effect was assigned to all observations deriving from the same sampling frame, and the variance of random effects was estimated with the restricted maximum likelihood estimator [29]. Omnibus tests of the regression models were used to examine correlations between survival and moderators, and Q tests to evaluate residual heterogeneity, i.e., to test whether residual variation was attributable to unobserved study characteristics [28]. For pairwise comparisons of survival and YLL, we set each phenotype as the reference group (intercept) in turn. Correction for multiple comparisons was not applied, as this study was not testing a proposition sensitive to incorrect rejections of null hypotheses [30].

\section{Results}

\section{Study Characteristics}

Eleven studies were North American [3, 7, 19, 31-38], eleven were Western European [8, 10, 39-47] and four were from Southeast and East Asia [5, 48-50]. One was multi-continental [4]. Twenty-two contributed data for calculating mean survival (1,340 subjects altogether) and twenty for YLL-mean. Mean age at onset in the twenty-two studies ranged 
from 45.0 to 67.5 years and the male proportion from 25 to $100 \%$. Eighteen contributed data for one FTD phenotype, and one study each provided data for two, three, four and five phenotypes. One study reported data for AD. Thus we had seven contributions for bvFTD, three for PNFA, two for SD, one for PPA, four for FTD-ALS, twelve for PSP and three for CBD. Six studies contributed data for median survival (1,497 subjects) and YLL-median. In these, the mean age at onset ranged from 56.2 to 66.2 years and the male proportion from 28.6 to $64.8 \%$. Three provided data on a single phenotype each, and one study each contributed to two, three and five phenotypes. One study also provided data for AD. Altogether, four studies contributed to median survival for bvFTD, two for PNFA, two for SD, three for FTD-ALS, one for PSP and one for PSP/CBD. Mean durations derived from descriptive analyses of deceased subjects, whereas median survival data derived from survival analyses.

\section{Survival and $Y L L$}

The aggregate mean survival (fig. 1) ranged from 2.50 years in FTD-ALS to 8.17 years in bvFTD, and aggregate median survival from 2.77 years in FTD-ALS to 12.22 years in SD (fig. 2). For $\mathrm{AD}$, mean and median survival were 6.86 and 11.95 years, respectively. YLL data are shown in figures 3 and 4. YLL-mean ranged from 9.78 years in PSP to 20.38 years in FTD-ALS, and YLL-median from 5.60 years in mixed PSP/CBD to 19.51 years in FTD-ALS. For AD, YLL were 14.32 and 1.63 years, respectively. Mean survival and YLL from analyses restricted to autopsy-validated samples were largely similar (see online suppl. table 2).

The aggregate median survival for FTD-ALS did not include data from one widely cited study [7] because those FTD-ALS subjects were represented in the bvFTD, PNFA and SD groups. A repeat analysis included the FTD-ALS data, but not the bvFTD data, to assess the robustness of the FTD-ALS survival estimate. The PNFA and SD data were entered since overlap with the FTD-ALS group was minimal - one of 27 PNFA subjects and one of 47 SD subjects. The median FTD-ALS survival thus derived was 2.88 years ( $95 \%$ CI $2.23-3.53$ ), close to the original estimate.

Omnibus tests showed that survival and YLL were associated with FTD phenotype in all models $(\mathrm{p}<0.001)$. $\mathrm{Q}$ tests for residual heterogeneity indicated that factors besides phenotype affected survival $(\mathrm{p}<0.01)$. The standard error of random effects for mean survival was 0.95 ( 0 in autopsy-validated samples) and 0.38 for median survival. We calculated the relative change in the variance of random effects when FTD phenotypes were added to a model with no fixed effects. Phenotypes accounted for $74.9 \%$ of the heterogeneity in mean survival and for $99.0 \%$ of that in median survival. In analyses restricted to autopsy-validated samples, phenotypes accounted for $100 \%$ of the heterogeneity, implying that unobserved moderators did not contribute to their within-phenotype variability.

Age (mean age at illness onset), sex (male proportion) and education (mean years) were analyzed in secondary models, separately and jointly (online suppl. table 3 ). Age and sex were not associated with survival in any models. Age was inversely associated with YLL-mean ( $\beta=$ $-0.55, p<0.001)$ and YLL-median $(\beta=-0.96, p<0.001)$. Male sex was inversely associated with YLL-median $(\beta=-0.21, p<0.001)$, but not with YLL-mean. With age and sex in the same model, the association of age with YLL-mean $(\beta=-0.61, \mathrm{p}<0.001)$ and YLL-median $(\beta=-1.09$, $\mathrm{p}<0.001$ ) held, but there were no associations for sex, suggesting that age mediated the earlier sex association. Education was associated with mean survival $(\beta=2.8, p=0.018)$ in analyses that could not be adjusted for age and sex due to insufficient data. It was also associated with YLL-median $(\beta=0.53, p=0.001)$, the latter enduring adjustment for age and sex $(\beta=0.74, p=0.022)$. 


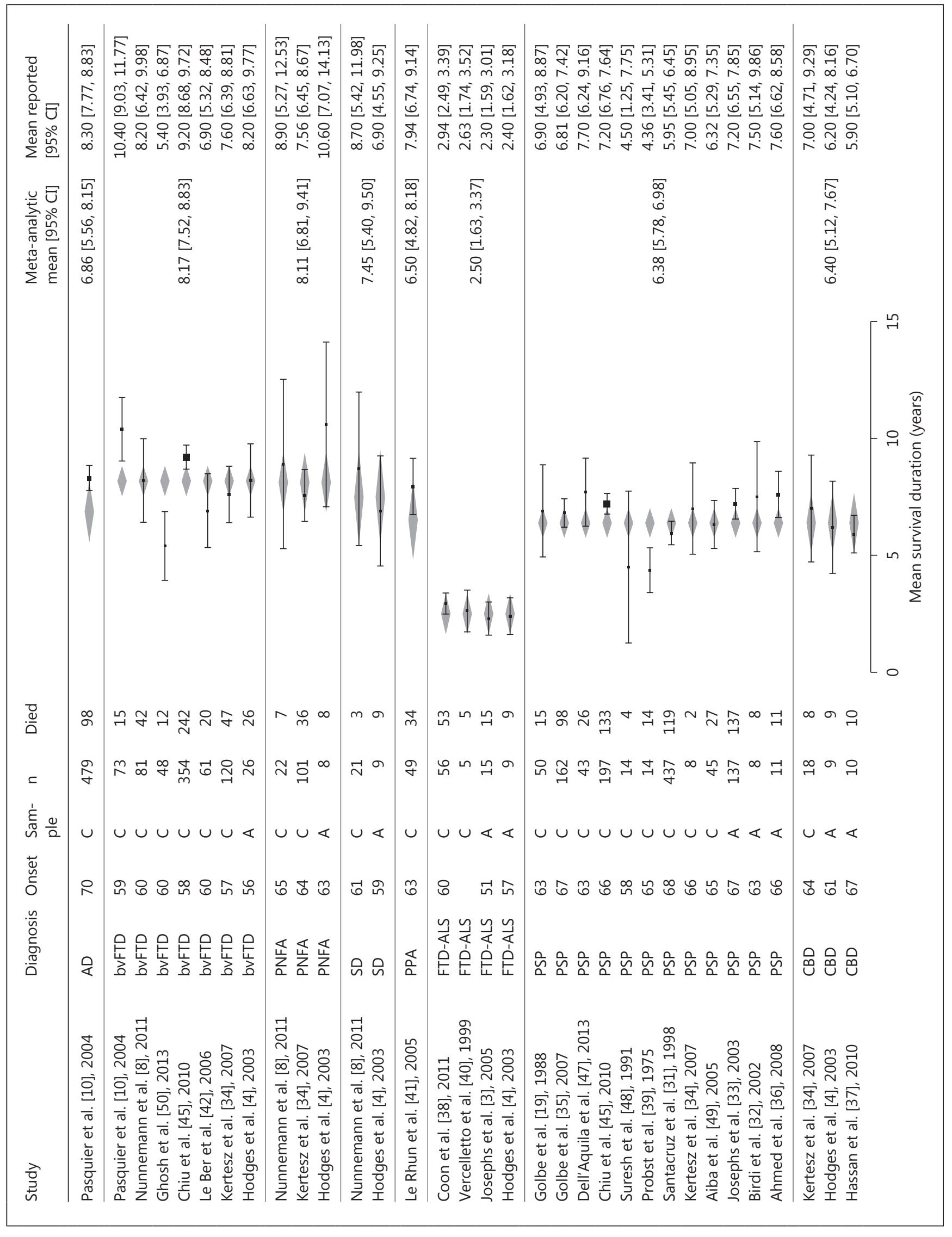




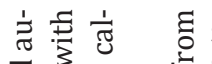

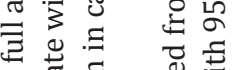

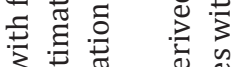

उ.

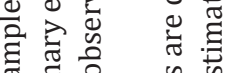

कह है छ के

II

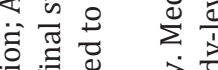

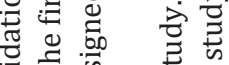

ए

के

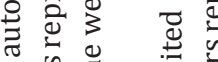

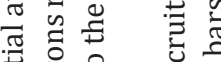

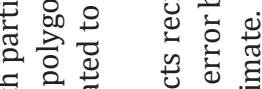

远焉焉

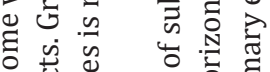

की

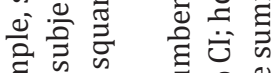

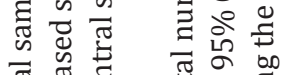

윰

II

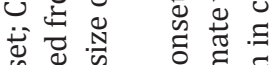

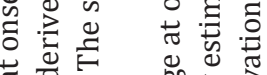

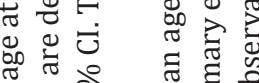

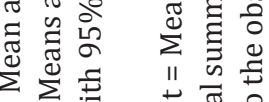

11 苛苛

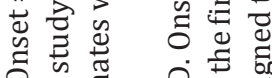

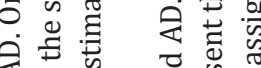

उ

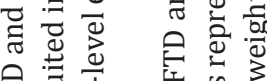

范宾空

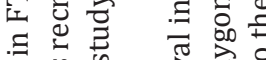

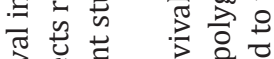

류 के

के के है

$\approx$ 宕

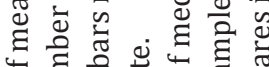

पे है

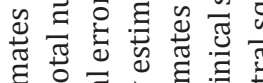

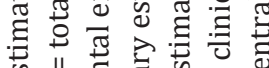

क्ष

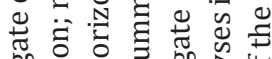

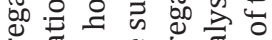

它:

\ن

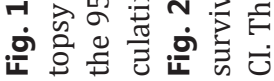




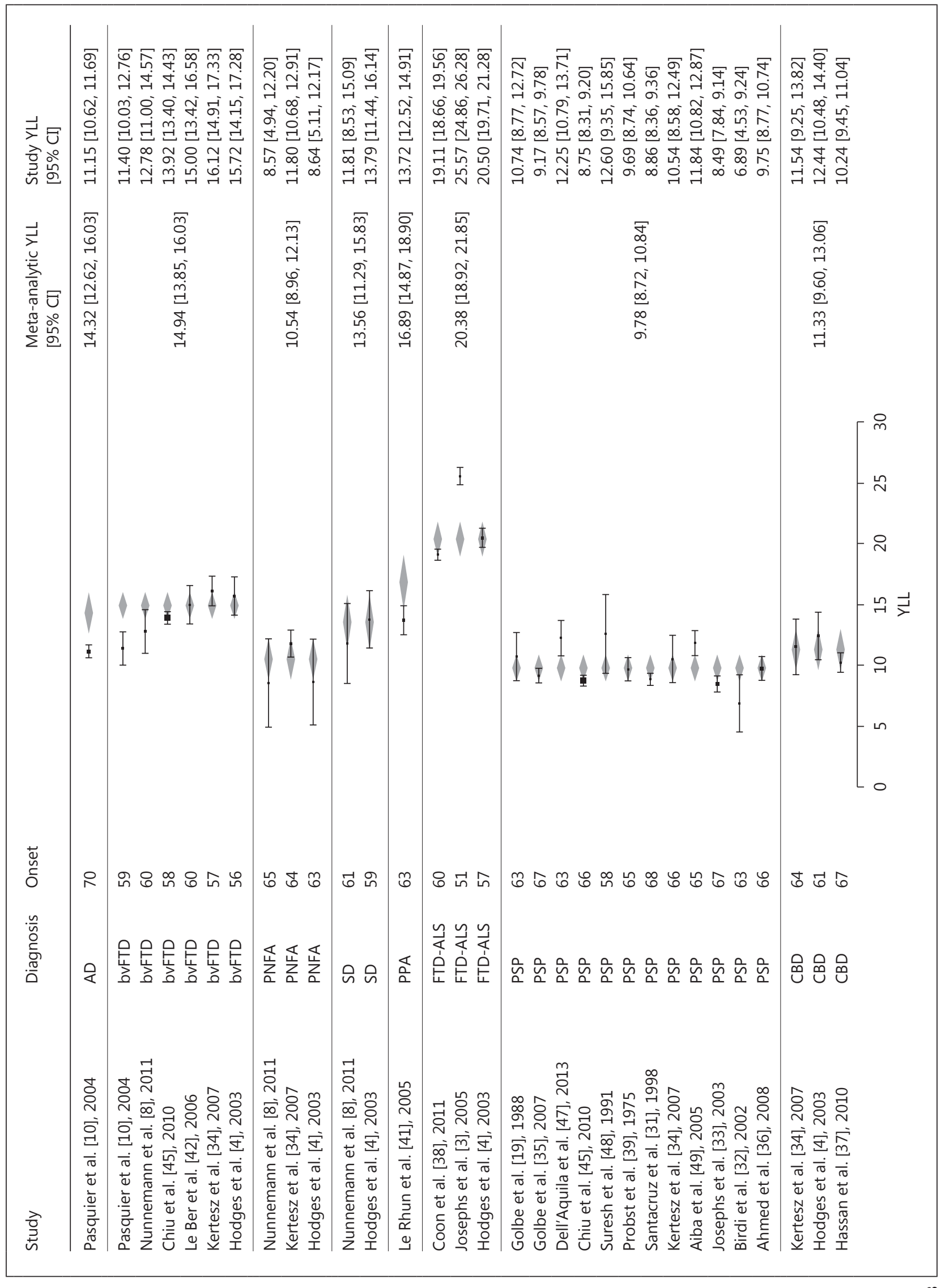




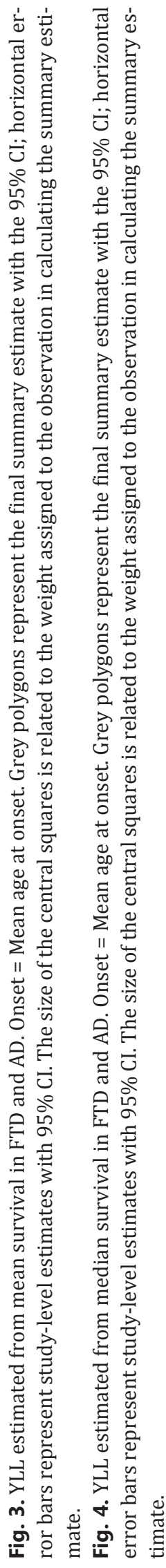




\section{Differences between FTD Phenotypes}

Pairwise comparisons (illustrated in online suppl. fig. 2) did not include mixed groups (PPA and PSP/CBD). FTD-ALS had the shortest mean and median survival. The bvFTD and PNFA groups had longer survival than the PSP and CBD groups. Differences in mean survival between SD, bvFTD and PNFA did not reach statistical significance. Median survival in SD was longer than in bvFTD, PNFA and PSP. PSP also had shorter median survival than bvFTD. Comparisons with AD were mixed: AD had shorter mean survival than bvFTD and longer median survival than bvFTD, PNFA and PSP. FTD-ALS had the largest YLL, followed by bvFTD. YLL-mean was higher in SD than in PNFA and PSP. AD had the shortest YLL-median, whereas its YLL-mean was intermediate.

\section{Discussion}

We used mixed effects modeling to derive aggregate estimates of FTD survival and premature mortality, demonstrating phenotype differences in FTD survival. We make several observations regarding these differences. Subjects who had FTD-ALS lived less than 3 years after illness onset and thus had the shortest life expectancy. The bvFTD and PNFA groups had the longest mean survival and SD the longest median survival. PSP and CBD generally had shorter survival than bvFTD, and PSP and CBD survival were close.

Only in the case of FTD-ALS was the FTD life expectancy shorter than that of AD. We recognize that the aggregate survival for AD reported here does not summarize all the existing data, since we culled only those collected alongside FTD data. However, our figures for FTD survival overlap the range reported for AD elsewhere [51,52]. A widely cited study comparing survival in pathologically defined FTD (i.e., irrespective of phenotype) with AD [9] reported shorter survival from the first clinical evaluation in the FTD subjects than in the AD group, but the sample did not explicitly exclude FTD-ALS subjects.

In contrast to survival, which emphasizes life expectancy, YLL highlights premature mortality. YLL is therefore useful for quantifying premature deaths in policy contexts. FTD-ALS, in accord with its short survival, had the largest YLL of the FTD phenotypes, and PNFA, PSP and CBD the lowest. Based on median survival, AD had shorter YLL than all FTD, which may be reflective of the older age at onset in the AD sample. However, as the YLL based on mean survival painted a different picture, comparisons of premature mortality between $\mathrm{AD}$ and FTD phenotypes (other than FTD-ALS) are still ambiguous. Larger YLL in FTD than in $\mathrm{AD}$ has been reported elsewhere [12], but the standardized mortality ratio (an age-corrected index of disease-related deaths) has been observed to be greater in AD than in FTD [53]. However, the standardized mortality ratio is highly sensitive to illness duration at diagnosis, and the latter study did not correct for it. It must also be acknowledged that the YLL are based on age means rather than distributions, and gender-weighted life expectancy is a partial correction used in lieu of the gender-specific survival we did not have. Thus the calculations are imperfect. It is unlikely, however, that this limitation meaningfully sways the results, since our observations fit the predictions of the age and survival data.

We accommodated more data and achieved a more complete characterization of FTD survival by conducting analyses of mean and median survival. This approach complicated the interpretation of comparisons, as some phenotypes (SD and $\mathrm{AD}$, in particular) differed substantively in the estimates of mean and median survival. The ready explanation is that studies included in the analysis of means and those in the analysis of medians differ in terms of their sampling methods and regional contexts. However, there is as well the intriguing prospect that mean-median differences within phenotypes are not mere artifacts and result instead from asymmetry in the underlying survival distributions. The survival of general 
populations in developed nations follows a distribution with a strong negative skew, with most deaths in late life, and a small fraction in youth and middle age [54]. One might hypothesize that for a disease with young- or mid-life onset and a sufficiently long course, most patients do not suffer from comorbidities that cause death within a few years. Therefore, most run the course of the disease with few dying prematurely, which causes survival to have a negative skew. In contrast, a positive skew is more likely when a disease has a very short course, as the outliers are those with unusually long survival. Such outliers have been observed in FTD-ALS [6]. The first implication of this idea is that mean and median survival should be measured in every natural history study, as meaningful differences may be present - and may facilitate identification of (modifiable) clinical or population factors that shape survival. A second implication is that hierarchies of phenotypes may differ with respect to mean versus median survival. These are hypotheses for future study.

Age at illness onset and sex were not associated with survival, and findings pertaining to education were indeterminate owing to limited data and contradictory associations. While most studies we examined did not find a link between survival and age $[4,7,8,10,22,42,43$, $46]$, sex $[4,7,8,22,38,42,43,46]$ or education $[7,8,46]$, some reported shorter survival with older age at onset $[5,19,35,45,47]$ and one with male sex [45]. One study found longer survival in males [55] and positive association between occupational attainment and survival (and not with education). Therefore our findings must be viewed as preliminary, since analyses of group means may miss trends seen in the individual studies. Further investigation is needed to clarify the contexts for associations between demographic characteristics and FTD survival.

We did not examine the effects of complicating features and comorbid conditions. Factors such as parkinsonism, dysphagia, swallowing apraxia, heart disease and diabetes mellitus have been ascertained in several studies $[5,7,8,19,38,42,48]$ that collectively did not yield enough to analyze here. So far, two studies have found no association between parkinsonism and survival $[7,42]$ and other factors have not been studied. An observation that most deaths $(68 \%)$ within 5 years of FTD onset resulted from pneumonia, cancer, cachexia, cardiovascular disease or accidents [8] lends credence to the idea that comorbid conditions hasten death. A cursory look at the causes of death reported in the literature - bronchopneumonia, choking, failure to thrive, circulatory system disorder, cerebrovascular disorder, head injury and carcinoma $[8,10,19,32,39,41,42,48,56]$ - also suggests that some deaths occur before the final stage.

FTD phenotypes accounted for most of the heterogeneity in mean survival, but $25 \%$ of the within-phenotype variability is unaccounted for. As residual heterogeneity was negligible in autopsy-validated samples, the first consideration was diagnostic misclassification, such as when psychiatric states are mistaken for FTD [57] or when ambiguity is caused by overlapping phenotypes [34] or atypical presentations [58]. However, misclassification seems unlikely to be large in this study since experts made the diagnoses. More objectively, just $1 \%$ of the heterogeneity was unexplained in the medians data, which derived from clinical rather than autopsy-validated samples. We propose two explanations for the lack of consistency in the values of heterogeneity. The first is temporal trends in diagnostic criteria. The publication year of studies that provided medians data is 2005 or later, whereas the means data start from 1975 (thus involving a larger variety of diagnostic criteria). These trends may have contributed to between-study and within-phenotype heterogeneity. The second is the paucity of studies with autopsy-validated samples and those providing medians (six studies in each case vs. twenty-two in the analysis of means). Estimation of residual heterogeneity is less precise in groups containing a small number of studies [29]. It is our suspicion that heterogeneity is underestimated in the autopsy-validated and medians groups and more reliable in the means analysis. Therefore, it is prudent to look beyond diagnostic error for sources of 
heterogeneity, such as mixing of phenotypes, complications and comorbid conditions, histopathological type of FTD, symptoms used to ascertain age at illness onset and population factors (encompassing ethnicity, diet, activity, health-seeking behavior, etc.). Four of the sixteen studies $(25.0 \%)$ that contributed means data did not explicitly exclude FTD-ALS; this fraction was smaller for studies contributing medians (one of the six, 16.7\%). Although described decades earlier, FTD and ALS co-occurrence did not become an important focus until the 2000s as genetics discoveries buttressed the clinical and histopathological evidence for an FTD-ALS syndrome $[59,60]$. Thus clinical studies from earlier decades usually did not distinguish the subset of bvFTD, PNFA and SD subjects who would today be classified as FTD-ALS. Furthermore, complex FTD phenotypes that cross syndrome boundaries are not uncommon [34]. It is worth noting that we directly adopted the diagnoses specified by the studies, which were sometimes those made at presentation $[4,8,41,50,56,61]$, sometimes the most recent $[10,32,33,36,46]$, and often had unspecified timing.

Individual clinical profiles are defined, besides the phenotype, by complicating features like dysphagia, autonomic dysfunction and parkinsonism, and comorbidities such as diabetes mellitus, heart disease and cancer - factors that, as noted earlier, may shape survival. Also, pathological diversity may contribute to within-phenotype differences, as the correspondence of FTD phenotypes with histopathological types [62] is imperfect. Two studies differed on how survival in tau-positive cases compares with that in tau-negative cases $[3,63]$, so the question remains open. At present, detecting a causal mutation, which applies to a minority of the cases, is the only reliable method for distinguishing histopathological type on clinical grounds. It is to be noted that our findings are not easily extrapolated to specific histopathological or genetic types and mainly reflect average survival in the phenotypes.

Determining the age at illness onset of an insidious disease is inherently subjective and, therefore, a contributor to error in measurement of survival. Individual factors that do not have group-level biases (such as those arising from the judgments of interviewed sources, or varying degrees of approximation in the age at illness onset derived from medical records) are captured in the study-level standard errors. As a result, they alter the weights assigned to the studies and the confidence intervals of the estimates, but do not increase residual heterogeneity. On the other hand, systematic biases in study design result in residual heterogeneity and may sway findings. For example, while most studies did not explicitly mention the symptoms used to define illness onset, one that specifically overlooked cognitive symptoms in a PSP sample [19] may have had lower 'true' ages at onset and, therefore, longer survival than that recorded in the study. In general, the various diagnostic criteria for bvFTD, PNFA, SD and AD focus on cognitive symptoms, while those for PSP and CBD focus on motor symptoms; as such, systematic biases may be expected in some studies. Furthermore, population factors such as cultural norms, health awareness and attitudes can systematically influence the ascertainment of illness onset and so the measurement of survival.

Population factors like diet, ethnicity and health seeking behavior have also been linked to the risk for developing dementia [64-66] and to dementia-related mortality [67, 68], although the research on these topics has largely focused on AD. Since our data derive largely from Caucasian subjects living in the United States and Western Europe, the generalizability of our findings to other regions might be at issue. The cross-cultural problem was raised in a report from India [50] that described florid presentations and called for modified criteria to accommodate cultural modes and facilitate earlier case identification, but there is also a report from Nigeria about a case presenting 2 years into the illness with features that matched contemporary diagnostic criteria [69].

In summary, FTD is, with the exception of FTD-ALS, a long illness, with its survival of 6-12 years similar to that of AD. The bvFTD, PNFA and SD phenotypes have comparable survival, apparently better than in PSP and CBD, but more research with prospective designs is needed 
for verification. In particular, studies of asymptomatic mutation carriers such as the Genetic Frontotemporal Dementia Initiative [70] (http://genfi.org.uk) and Advancing Research and Treatment for Frontotemporal Lobar Degeneration (ARTFL) (https://clinicaltrials.gov/ct2/ show/NCT02365922) offer opportunities for observing transitions from asymptomatic status to subclinical and clinical phases and for characterizing survival. Within-phenotype mean-median differences in survival can arise from study differences, but asymmetry in the underlying survival distributions is possible and merits study. Such asymmetry may reflect the distribution of the population factors, complicating features and comorbid conditions that may shape survival through earlier deaths, or of the characteristics that may provide resilience (such as in FTD-ALS with longer survival). The effects of these and other potential moderators of survival are pressing questions for defining covariates for natural history studies and clinical trials and, where modifiable, for developing tailored interventions.

\section{Acknowledgements}

This research was supported by the Johns Hopkins Alzheimer's Disease Research Center (NIA grant P50AG05146), the Jane Tanger Black Fund for Young-Onset Dementia Research, the Robert and Nancy Hall family, the Samuel I. Newhouse Foundation and the Richman Family professorship. Special thanks go to Florence Pasquier, MD, PhD (Centre Hospitalier Régional Universitaire de Lille, Lille, France) and Janine Diehl-Schmid, MD (Technische Universität München, Munich, Germany), who generously provided original data from their studies $[8,10]$, to Shunichiro Shinagawa, MD, PhD (Jikei University School of Medicine, Tokyo, Japan), who translated a Japanese study [49], and to Somya Abubucker, BA, who assisted us with editing.

\section{Disclosure Statement}

Kalyani Kansal is supported by a scholarship from the Jane Tanger Black Fund for Young-Onset Dementia Research. Peter V. Rabins has given legal testimony for Janssen Pharmaceutica and is supported by grants from the NIA and NIMH, the Richman Family Professorship, the Robert and Nancy Hall family and the Samuel Newhouse Foundation. Chiadi U. Onyike has been a consultant to Pfizer, a clinical investigator in trials sponsored by Forest Pharmaceuticals, GE Healthcare and TauRx, and is supported by grants from the NIA and NINDS, the Robert and Nancy Hall family and the Jane Tanger Black Fund for Young-Onset Dementia Research. Manisha Mareddy, Kelly L. Sloane, Alexa A. Minc and John B. McGready report no disclosures.

\section{References}

1 Gorno-Tempini ML, Hillis AE, Weintraub S, Kertesz A, Mendez M, Cappa SF, et al: Classification of primary progressive aphasia and its variants. Neurology 2011;76:1006-1014.

2 Onyike CU: What is the life expectancy in frontotemporal lobar degeneration? Neuroepidemiology 2011;37: 166-167.

3 Josephs KA, Knopman DS, Whitwell JL, Boeve BF, Parisi JE, Petersen RC, et al: Survival in two variants of taunegative frontotemporal lobar degeneration: FTLD-U vs FTLD-MND. Neurology 2005;65:645-647.

4 Hodges JR, Davies R, Xuereb J, Kril J, Halliday G: Survival in frontotemporal dementia. Neurology 2003;61: 349-354.

5 Kang SJ, Cha KR, Seo SW, Kim EA, Cheong HK, Kim EJ, et al: Survival in frontotemporal lobar degeneration in a Korean population. Alzheimer Dis Assoc Disord 2010;24:339-342.

6 Hu WT, Seelaar H, Josephs KA, Knopman DS, Boeve BF, Sorenson EJ, et al: Survival profiles of patients with frontotemporal dementia and motor neuron disease. Arch Neurol 2009;66:1359-1364.

7 Roberson ED, Hesse JH, Rose KD, Slama H, Johnson JK, Yaffe K, et al: Frontotemporal dementia progresses to death faster than Alzheimer disease. Neurology 2005;65:719-725.

8 Nunnemann S, Last D, Schuster T, Förstl H, Kurz A, Diehl-Schmid J: Survival in a German population with frontotemporal lobar degeneration. Neuroepidemiology 2011;37:160-165.

9 Rascovsky K, Salmon DP, Lipton AM, Leverenz JB, DeCarli C, Jagust WJ, et al: Rate of progression differs in frontotemporal dementia and Alzheimer disease. Neurology 2005;65:397-403. 
10 Pasquier F, Richard F, Lebert F: Natural history of frontotemporal dementia: comparison with Alzheimer's disease. Dement Geriatr Cogn Disord 2004;17:253-257.

11 Koedam ELGE, Pijnenburg YAL, Deeg DJH, Baak MME, van der Vlies AE, Scheltens P, et al: Early-onset dementia is associated with higher mortality. Dement Geriatr Cogn Disord 2008;26:147-152.

12 Brodaty H, Seeher K, Gibson L: Dementia time to death: a systematic literature review on survival time and years of life lost in people with dementia. Int Psychogeriatr 2012;24:1034-1045.

13 Rascovsky K, Hodges JR, Knopman D, Mendez MF, Kramer JH, Neuhaus J, et al: Sensitivity of revised diagnostic criteria for the behavioural variant of frontotemporal dementia. Brain 2011;134:2456-2477.

14 Neary D, Snowden JS, Gustafson L, Passant U, Stuss D, Black S, et al: Frontotemporal lobar degeneration: a consensus on clinical diagnostic criteria. Neurology 1998;51:1546-1554.

15 Clinical and neuropathological criteria for frontotemporal dementia. The Lund and Manchester Groups. J Neurol Neurosurg Psychiatry 1994;57:416-418.

16 Mesulam MM: Primary progressive aphasia - differentiation from Alzheimer's disease. Ann Neurol 1987;22: 533-534.

17 Mesulam MM: Primary progressive aphasia. Ann Neurol 2001;49:425-432.

18 Steele JC, Richardson JC, Olszewski J: Progressive supranuclear palsy: a heterogeneous degeneration involving the brain stem, basal ganglia and cerebellum with vertical gaze and pseudobulbar palsy, nuchal dystonia and dementia. Semin Neurol 2014;34:129-150.

19 Golbe LI, Davis PH, Schoenberg BS, Duvoisin RC: Prevalence and natural history of progressive supranuclear palsy. Neurology 1988;38:1031-1034.

20 Rajput AH, Rozdilsky B, Rajput A: Accuracy of clinical diagnosis in parkinsonism - a prospective study. Can J Neurol Sci 1991;18:275-278.

21 Rajput AH, Rozdilsky B, Ang L, Rajput A: Significance of parkinsonian manifestations in essential tremor. Can J Neurol Sci 1993;20:114-117.

22 Litvan I, Mangone CA, McKee A, Verny M, Parsa A, Jellinger K, et al: Natural history of progressive supranuclear palsy (Steele-Richardson-Olszewski syndrome) and clinical predictors of survival: a clinicopathological study. J Neurol Neurosurg Psychiatry 1996;60:615-620.

23 Rebeiz JJ, Kolodny EH, Richardson EP: Corticodentatonigral degeneration with neuronal achromasia. Arch Neurol 1968;18:20-33.

24 Gibb WR, Luthert PJ, Marsden CD: Corticobasal degeneration. Brain 1989;112:1171-1192.

25 Litvan I, Agid Y, Goetz C, Jankovic J, Wenning GK, Brandel JP, et al: Accuracy of the clinical diagnosis of corticobasal degeneration: a clinicopathologic study. Neurology 1997;48:119-125.

26 Brooks BR, Miller RG, Swash M, Munsat TL; World Federation of Neurology Research Group on Motor Neuron Diseases: El Escorial revisited: revised criteria for the diagnosis of amyotrophic lateral sclerosis. Amyotroph Lateral Scler Other Motor Neuron Disord 2000;1:293-299.

27 Strong MJ, Grace GM, Freedman M, Lomen-Hoerth C, Woolley S, Goldstein LH, et al: Consensus criteria for the diagnosis of frontotemporal cognitive and behavioural syndromes in amyotrophic lateral sclerosis. Amyotroph Lateral Scler 2009;10:131-146.

28 Viechtbauer W: Conducting meta-analyses in R with the metafor package. J Stat Softw 2010;36:1-48.

29 Viechtbauer W: Bias and efficiency of meta-analytic variance estimators in the random-effects model. J Educ Behav Stat 2005;30:261-293.

30 Benjamini Y, Hochberg Y: Controlling the false discovery rate: a practical and powerful approach to multiple testing. J R Stat Soc Series B Stat Methodol 1995;57:289-300.

31 Santacruz P, Uttl B, Litvan I, Grafman J: Progressive supranuclear palsy: a survey of the disease course. Neurology 1998;50:1637-1647.

32 Birdi S, Rajput AH, Fenton M, Donat JR, Rozdilsky B, Robinson C, et al: Progressive supranuclear palsy diagnosis and confounding features: report on 16 autopsied cases. Mov Disord 2002;17:1255-1264.

33 Josephs KA, Dickson DW: Diagnostic accuracy of progressive supranuclear palsy in the Society for Progressive Supranuclear Palsy brain bank. Mov Disord 2003;18:1018-1026.

34 Kertesz A, Blair M, McMonagle P, Munoz DG: The diagnosis and course of frontotemporal dementia. Alzheimer Dis Assoc Disord 2007;21:155-163.

35 Golbe LI, Ohman-Strickland PA: A clinical rating scale for progressive supranuclear palsy. Brain 2007;130: 1552-1565.

36 Ahmed Z, Josephs KA, Gonzalez J, DelleDonne A, Dickson DW: Clinical and neuropathologic features of progressive supranuclear palsy with severe pallido-nigro-luysial degeneration and axonal dystrophy. Brain 2008;131:460-472.

37 Hassan A, Whitwell JL, Boeve BF, Jack CR, Parisi JE, Dickson DW, et al: Symmetric corticobasal degeneration (S-CBD). Parkinsonism Relat Disord 2010;16:208-214.

38 Coon EA, Sorenson EJ, Whitwell JL, Knopman DS, Josephs KA: Predicting survival in frontotemporal dementia with motor neuron disease. Neurology 2011;76:1886-1893.

39 Probst A, Dufresne JJ: Paralysie supranucléaire progressive (ou dystonie oculo-facio-cervicale). Schweiz Arch Neurol Neurochir Psychiatr 1975;116:107-134.

40 Vercelletto M, Ronin M, Huvet M, Magne C, Feve JR: Frontal type dementia preceding amyotrophic lateral sclerosis: a neuropsychological and SPECT study of five clinical cases. Eur J Neurol 1999;6:295-299. 
41 Le Rhun E, Richard F, Pasquier F: Natural history of primary progressive aphasia. Neurology 2005;65:887891.

42 Le Ber I, Guedj E, Gabelle A, Verpillat P, Volteau M, Thomas-Antérion C, et al: Demographic, neurological and behavioural characteristics and brain perfusion SPECT in frontal variant of frontotemporal dementia. Brain 2006;129:3051-3065.

43 Garcin B, Lillo P, Hornberger M, Piguet O, Dawson K, Nestor PJ, et al: Determinants of survival in behavioral variant frontotemporal dementia. Neurology 2009;73:1656-1661.

44 Lillo P, Garcin B, Hornberger M, Bak TH, Hodges JR: Neurobehavioral features in frontotemporal dementia with amyotrophic lateral sclerosis. Arch Neurol 2010;67:826-830.

45 Chiu WZ, Kaat LD, Seelaar H, Rosso SM, Boon AJ, Kamphorst W, et al: Survival in progressive supranuclear palsy and frontotemporal dementia. J Neurol Neurosurg Psychiatry 2010;81:441-445.

46 Hodges JR, Mitchell J, Dawson K, Spillantini MG, Xuereb JH, McMonagle P, et al: Semantic dementia: demography, familial factors and survival in a consecutive series of 100 cases. Brain 2010;133:300-306.

47 dell'Aquila C, Zoccolella S, Cardinali V, de Mari M, Iliceto G, Tartaglione B, et al: Predictors of survival in a series of clinically diagnosed progressive supranuclear palsy patients. Parkinsonism Relat Disord 2013;19:980-985.

48 Suresh TG, Rao TV: Progressive supranuclear palsy. Report of 14 cases with special reference to unusual features. J Assoc Physicians India 1991;39:471-475.

49 Aiba I, Saito Y, Tamakoshi A, Matsuoka Y: Prognosis of patients with progressive supranuclear palsy. Rinsho Shinkeigaku 2005;45:565-570.

50 Ghosh A, Dutt A, Ghosh M, Bhargava P, Rao S: Using the revised diagnostic criteria for frontotemporal dementia in India: evidence of an advanced and florid disease. PLoS One 2013;8:e60999.

51 Ganguli M, Dodge HH, Shen C, Pandav RS, DeKosky ST: Alzheimer disease and mortality: a 15-year epidemiological study. Arch Neurol 2005;62:779-784.

52 Waring SC, Doody RS, Pavlik VN, Massman PJ, Chan W: Survival among patients with dementia from a large multi-ethnic population. Alzheimer Dis Assoc Disord 2005;19:178-183.

53 Nilsson K, Gustafson L, Hultberg B: Survival in a large elderly population of patients with dementia and other forms of psychogeriatric diseases. Dement Geriatr Cogn Disord 2011;32:342-350.

54 Wilmoth JR, Horiuchi S: Rectangularization revisited: variability of age at death within human populations. Demography 1999;36:475-495.

55 Massimo L, Zee J, Xie SX, McMillan CT, Rascovsky K, Irwin DJ, et al: Occupational attainment influences survival in autopsy-confirmed frontotemporal degeneration. Neurology 2015;84:2070-2075.

56 Goetz CG, Leurgans S, Lang AE, Litvan I: Progression of gait, speech and swallowing deficits in progressive supranuclear palsy. Neurology 2003;60:917-922.

57 Woolley JD, Khan BK, Murthy NK, Miller BL, Rankin KP: The diagnostic challenge of psychiatric symptoms in neurodegenerative disease: rates of and risk factors for prior psychiatric diagnosis in patients with early neurodegenerative disease. J Clin Psychiatry 2011;72:126-133.

58 Velakoulis D, Walterfang M, Mocellin R, Pantelis C, McLean C: Frontotemporal dementia presenting as schizophrenia-like psychosis in young people: clinicopathological series and review of cases. Br J Psychiatry 2009; 194:298-305.

59 Morita M, Al-Chalabi A, Andersen PM, Hosler B, Sapp P, Englund E, et al: A locus on chromosome 9p confers susceptibility to ALS and frontotemporal dementia. Neurology 2006;66:839-844.

60 Vance C, Al-Chalabi A, Ruddy D, Smith BN, Hu X, Sreedharan J, et al: Familial amyotrophic lateral sclerosis with frontotemporal dementia is linked to a locus on chromosome 9p13.2-21.3. Brain 2006;129:868-876.

61 Kertesz A, Munoz DG: Primary progressive aphasia and Pick complex. J Neurol Sci 2003;206:97-107.

62 Boeve BF: Links between frontotemporal lobar degeneration, corticobasal degeneration, progressive supranuclear palsy, and amyotrophic lateral sclerosis. Alzheimer Dis Assoc Disord 2007;21:S31-S38.

63 Xie SX, Forman MS, Farmer J, Moore P, Wang Y, Wang X, et al: Factors associated with survival probability in autopsy-proven frontotemporal lobar degeneration. J Neurol Neurosurg Psychiatry 2008;79:126-129.

64 Larson EB, Wang L, Bowen JD, McCormick WC, Teri L, Crane P, et al: Exercise is associated with reduced risk for incident dementia among persons 65 years of age and older. Ann Intern Med 2006;144:73-81.

65 Barberger-Gateau P, Raffaitin C, Letenneur L, Berr C, Tzourio C, Dartigues JF, et al: Dietary patterns and risk of dementia: the Three-City cohort study. Neurology 2007;69:1921-1930.

66 Wang HX, Karp A, Winblad B, Fratiglioni L: Late-life engagement in social and leisure activities is associated with a decreased risk of dementia: a longitudinal study from the Kungsholmen project. Am J Epidemiol 2002; 155:1081-1087.

67 Mehta KM, Yaffe K, Pérez-Stable EJ, Stewart A, Barnes D, Kurland BF, et al: Race/ethnic differences in AD survival in US Alzheimer's Disease Centers. Neurology 2008;70:1163-1170.

68 Lanska DJ: Dementia mortality in the United States. Results of the 1986 National Mortality Followback Survey. Neurology 1998;50:362-367.

69 Akinyemi RO, Owolabi MO, Makanjuola VA, Ogunseyinde AO, Ogunniyi A: Frontotemporal dementia in a Nigerian woman: case report and brief review of the literature. Afr J Med Med Sci 2009;38:71-75.

70 Rohrer JD, Warren JD, Fox NC, Rossor MN: Presymptomatic studies in genetic frontotemporal dementia. Rev Neurol (Paris) 2013;169:820-824. 